UPR-0502T

\title{
COORDINATE AND KÄHLER $\sigma$-MODEL ANOMALIES AND THEIR CANCELLATION IN STRING EFFECTIVE FIELD THEORIES
}

\author{
Gabriel Lopes Cardoso \\ and \\ Burt A. Ovrutf \\ Department of Physics \\ University of Pennsylvania \\ Philadelphia, PA 19104-6396
}

\begin{abstract}
We discuss the complete set of one-loop triangle graphs involving the Yang-Mills gauge connection, the Kähler connection and the $\sigma$-model coordinate connection in the effective field theory of $(2,2)$ symmetric $Z_{N}$ orbifolds. That is, we discuss pure gauge, pure Kähler and pure $\sigma$-model coordinate anomalies as well as the mixed anomalies, such as Kähler-gauge, some of which have been discussed elsewhere. We propose a mechanism for restoring both Kähler and $\sigma$-model coordinate symmetry based upon the introduction of two types of counterterms. Finally, we enlarge the $\sigma$-model generalization of the Green-Schwarz mechanism to allow the removal of the universal parts of a wider class of anomalies than those previously discussed.
\end{abstract}

\footnotetext{
${ }^{1}$ Work supported in part by the Department of Energy under Contract No. DOE-AC02-76-ERO3071 and NATO Grant No. 860684.
} 


\section{Introduction}

Kähler superspace provides a powerful and elegant tool for the description of the $N=1$ supergravity-matter system [1] in four dimensions. The structure group of Kähler superspace contains an abelian group, denoted $U_{K}(1)$, in addition to the Lorentz group. By construction, $N=1$ supergravity-matter systems are invariant under Kähler transformations. These consist, in part, of field dependent $U_{K}(1)$ transformations of chiral component field fermions. In addition to the Kähler symmetry, the tree-level supergravity-matter theory possesses several other invariances. A generic matter manifold with Kähler metric $g_{i \bar{j}}$ is parameterized by chiral matter fields $\phi^{i}$ and $\bar{\phi}^{\bar{j}}[2]$. Under $\sigma$ - model general coordinate transformations of these fields, the tree-level Lagrangian and, hence, the action transform like scalars. It follows that the partition function is, naively, invariant under $\sigma$-model coordinate transformations. Under certain circumstances, the isometries of the Kähler metric $g_{i \bar{j}}$ may be actual symmetries of the tree-level component Lagrangian. Another invariance of relevance for this paper is the invariance under Yang-Mills transformations. Hence, the covariant derivative $\mathcal{D}_{m}$ of a generic matter Weyl spinor $\chi_{\alpha}^{i}$ contains connections $a_{m}, \Gamma^{i}{ }_{j k}$ and $v_{m}^{(r)}$ for gauging Kähler transformations, $\sigma$-model coordinate transformations and Yang-Mills gauge transformations, respectively. We will not discuss space-time Lorentz transformations and, hence, we will not consider the Lorentz connection $\omega_{m}{ }^{\alpha}{ }_{\beta}$ in the main part of this paper. Mixed $\sigma$-model anomalies involving this connection have been considered in a recent paper [3], and we will briefly summarize and expand these results in Appendix A. Given these three symmetries at tree-level, one might ask whether or not they are anomalous at the one-loop level. As always in four dimensions, one-loop fermionic triangle graphs are potentially anomalous. In such a triangle graph massless fermions run around the loop and any of the three gauge connections under consideration, $a, \Gamma$, and $v$, can couple to each of three vertices of the triangle graph. Hence, the symmetries under consideration can be broken by pure aaa, ГГГ and $v v v$ graphs as well as by any of the mixed graphs, such as avv or $\Gamma v v$. In this paper, we will focus on $(2,2)$ symmetric $Z_{N}$ orbifolds [4,5]. The effective theory of these orbifolds is, by construction [4], free of pure Yang-Mills gauge anomalies, pure Lorentz anomalies and mixed Yang-Mills, Lorentz anomalies. Therefore, we need not consider the pure 
vvv graph in our discussion. One has, however, to worry about anomalies in Kähler and $\sigma$-model coordinate transformations. Anomalies in $\sigma$-model coordinate transformations, as computed from ГГГ graphs, have been studied in other contexts in [6]. Recently, much attention has been devoted to the study of the two mixed graphs avv and $\Gamma v v$, in the context of non-harmonic gauge coupling constants $[3,7,8,9,10]$. It was shown $[3,8,9]$ that these two mixed graphs contribute non-local terms to the 1-loop effective Lagrangian, which are not invariant under either Kähler transformations or under modular transformations (a subset of isometry transformations). Clearly, it is not enough to only consider the two mixed $a v v$ and $\Gamma v v$ graphs when discussing the issue of anomalies under Kähler and $\sigma$-model coordinate transformations. In this paper, we will compute all anomalous contributions, from both the pure aaa and ГГГ graphs as well as from all the mixed graphs, such as avv and $\Gamma v v$. We will find that all these graphs contribute non-local terms to the 1-loop effective Lagrangian, which either break Kähler invariance or transform as densities under $\sigma$-model coordinate transformations, and, hence, are anomalous under these transformations. That is, the 1-loop effective Lagrangian, as computed from the massless fields in the $Z_{N}$ orbifold theory, breaks Kähler invariance and is also not a scalar under $\sigma$-model coordinate transformations. This state of affairs is devastating to making physical sense of orbifold theories. It follows that one would like to devise some cure to render such theories anomaly free. It has been shown in explicit string amplitude calculations [7] that, in the case of mixed $a v v$ and $\Gamma v v$ graphs, the integration over heavy orbifold states contributes local counterterms to the 1-loop effective Lagrangian of the light fields, so as to restore modular invariance of the gauge coupling constants [9]. These explicit string calculations then suggest a general mechanism, based upon the introduction of heavy state induced counterterms, for rendering the theory anomaly free. Hence, we will follow a similar strategy and postulate the existence of two types of counterterms for the restoration of invariance of the partition function under Kähler transformations and under $\sigma$-model coordinate transformations. The first type of counterterm will be added to the anomalous effective 1-loop Lagrangian so as to restore invariance under Kähler transformations. This counterterm is of the type of a superpotential term for the untwisted moduli and the dilaton. A second type of counterterms will be added to the anomalous Lagrangian so as to render the effective Lagrangian a scalar under $\sigma$-model 
coordinate transformations. In order to fix the explicit form of all these counterterms we will choose the standard coordinate system for parametrizing the manifold of the untwisted moduli and the dilaton. In the standard coordinate system, superstring amplitudes are expected to be modular invariant [11]. Hence, the effective theory for orbifolds should be modular invariant as well. By demanding modular invariance we will be able to uniquely determine the moduli dependence of the superpotential term as well as of the type two counterterms. Additional computations of three particle scattering string amplitudes, such as three dilaton amplitudes, would be required in order to determine the dilaton dependence of the counterterms. Finally, it has been shown [10] that the universal part of the mixed $a v v$ and $\Gamma v v$ anomalies gets removed by a $\sigma$-model generalization [8] of the Green-Schwarz mechanism [12] involving the dilaton, dilatino, axion linear supermultiplet. We will close this paper by discussing an extension of this mechanism. Specifically we will show that the universal part of the pure Kähler anomaly, aaa, can, in principle, be removed as well by a Green-Schwarz type mechanism. However, only an explicit calculation of the relevant three point correlation function in string theory can clarify whether or not a part of the pure Kähler anomaly (and how much of it) is removed by this mechanism in string theory.

\section{2. $\mathrm{U}_{\mathrm{K}}(\mathbf{1})$ Superspace}

Kähler superspace provides a powerful and elegant tool for the description of the supergravity-matter system [1]. We begin by recalling here some of the features of Kähler superspace geometry which will be relevant in the subsequent discussion. A complete description of the properties of $U_{K}(1)$ superspace can be found in [1]. The structure group of Kähler superspace is taken to be $S L(2, \mathcal{C}) \times U_{K}(1)$ and, accordingly, one introduces two Lie algebra valued one-form gauge connections $\phi_{B}{ }^{A}=d z^{M} \phi_{M B}{ }^{A}$ and $A=d z^{M} A_{M}$ corresponding to the Lorentz and $U_{K}(1)$ groups respectively. In addition, one introduces a supervielbein $E_{M}{ }^{A}$ and the associated one-forms $E^{A}=$ $d z^{M} E_{M}{ }^{A}$. The $U_{K}(1)$ gauge connection $A$ is a composite gauge connection defined by

$$
\begin{aligned}
A_{\alpha} & =\frac{1}{4} \mathcal{D}_{\alpha} K \\
A^{\dot{\alpha}} & =-\frac{1}{4} \overline{\mathcal{D}}^{\dot{\alpha}} K
\end{aligned}
$$




$$
A_{\alpha \dot{\alpha}}=-\frac{i}{8}\left[\mathcal{D}_{\alpha}, \overline{\mathcal{D}}_{\dot{\alpha}}\right] K
$$

where the prepotential $K\left(\Phi_{i}, \Phi_{i}^{+}\right)$is the Kähler potential for matter fields. All matter superfields have vanishing $U_{K}(1)$ weight, $\omega\left(\Phi_{i}\right)=0$. Under a Kähler transformation

$$
K\left(\Phi_{i}, \Phi_{i}^{+}\right) \rightarrow K\left(\Phi_{i}, \Phi_{i}^{+}\right)+F\left(\Phi_{i}\right)+\bar{F}\left(\Phi_{i}^{+}\right)
$$

the one-form $A$ transforms as

$$
A \rightarrow A+\frac{i}{2} d \operatorname{ImF}
$$

Also, under a Kähler transformation the supervielbein one-forms $E^{A}$ can be shown [1] to transform as

$$
E^{A} \rightarrow E^{A} \exp \left[-\frac{i}{2} \omega\left(E^{A}\right) \operatorname{Im} F\right]
$$

where

$$
\omega\left(E^{\alpha}\right)=1, \omega\left(E_{\dot{\alpha}}\right)=-1, \omega\left(E^{a}\right)=0
$$

Solving the Bianchi identities subject to a set of natural constraints [13], one finds that all components of the torsion and curvature may be expressed in terms of a set of superfields and their coordinate derivatives:

$$
\begin{array}{cccccc}
\text { superfield } & R & R^{+} & G_{\alpha \dot{\alpha}} & W_{\alpha \beta \gamma}, X_{\alpha} & \bar{W}_{\dot{\alpha} \dot{\beta} \dot{\gamma}}, \bar{X}_{\dot{\alpha}} \\
U_{K}(1) \text { weight } & 2 & -2 & 0 & 1 & -1
\end{array}
$$

where

$$
\begin{aligned}
& X_{\alpha}=\mathcal{D}_{\alpha} \mathcal{R}-\overline{\mathcal{D}}^{\dot{\alpha}} G_{\alpha \dot{\alpha}}=-\frac{\kappa^{2}}{8}\left(\overline{\mathcal{D}}^{2}-8 R\right) \mathcal{D}_{\alpha} K \\
& \bar{X}^{\dot{\alpha}}=\overline{\mathcal{D}}^{\dot{\alpha}} R^{+}+\mathcal{D}_{\alpha} G^{\alpha \dot{\alpha}}=-\frac{\kappa^{2}}{8}\left(\mathcal{D}^{2}-8 R^{+}\right) \overline{\mathcal{D}}^{\dot{\alpha}} K
\end{aligned}
$$

and $\kappa^{2}=8 \pi M_{P}^{-2}$, where $M_{P}$ is the Planck mass. $X_{\alpha}$ is the superfield fieldstrength of the $U_{K}(1)$ gauge connection.

\section{Classical Symmetries of $(2,2)$ Symmetric Orbifold Lagrangians}

In this section, we will consider $(2,2)$ symmetric $Z_{N}$ orbifolds [4,5] with gauge group $G=E_{8} \otimes E_{6} \otimes U(1)^{2}$. These are the orbifolds which have been discussed most 
extensively in the context of non-harmonic gauge coupling constants in superstring theories. We will, for simplicity, furthermore restrict our discussion to the subset of these orbifolds which do not contain $(1,2)$ moduli; that is, to $Z_{7}, Z_{8}$ and $Z_{12^{\prime}}$. Our considerations can, however, be generalized in a straightforward way to the case of the remaining $E_{8} \otimes E_{6} \otimes U(1)^{2}$ orbifolds, $Z_{6^{\prime}}, Z_{8^{\prime}}$ and $Z_{12}$, which do contain $(1,2)$ moduli. Generically, the spectrum [4] of the orbifolds under consideration contains three uncharged untwisted $(1,1)$ moduli, $T^{I}$, as well as untwisted and twisted 27 matter fields, which we will be collectively denoting by $\phi^{i}$. We also consider twisted $(1,1)$ moduli, denoted $C_{d}$. However we will ignore matter fields which are singlets under the $E_{6}$, since the structure of their Kähler potentials is unknown. In addition to the above fields, we will also discuss the dilaton field $S$ [14]. Each of the untwisted $(1,1)$ moduli $T^{I}$ parametrizes a coset space $S U(1,1) / U(1) \simeq S L(2, \mathcal{R}) / U(1)$ with isometry group $S L(2, \mathcal{R})[15]$. The Kähler potential for the moduli and matter fields under consideration reads $[5,16]$

$$
\begin{aligned}
K_{M}= & \kappa^{-2} \sum_{I}-\ln (T+\bar{T})^{I}+\sum_{i} e^{\ln \mathcal{G}_{i}} \bar{\phi}^{i} e^{V} \phi^{i} \\
& +\sum_{d} e^{\ln \mathcal{G}_{d}} \bar{C}_{d} e^{V} C_{d}+\mathcal{O}\left((\bar{\phi} \phi)^{2}\right)+\mathcal{O}\left((\bar{C} C)^{2}\right)
\end{aligned}
$$

where

$$
\begin{aligned}
\mathcal{G}_{i}\left(T^{I}, \bar{T}^{I}\right) & =\prod_{I}\left[T^{I}+\bar{T}^{I}\right]^{-q_{i}^{I}} \\
\mathcal{G}_{d}\left(T^{I}, \bar{T}^{I}\right) & =\prod_{I}\left[T^{I}+\bar{T}^{I}\right]^{-q_{d}^{I}}
\end{aligned}
$$

The exponents $q_{i}^{I}$ and $q_{d}^{I}$ are associated with the matter fields $\phi^{i}$ and twisted moduli fields $C_{d}$, respectively [16]. The Kähler potential for the universal dilaton supermultiplet, present in any compactification scheme of the heterotic superstring theory, is given by [14]

$$
K_{S}=-\kappa^{-2} \ln (S+\bar{S})
$$

where $S$ is the chiral representation of the dilaton. We have chosen to normalize the fields $T^{I}$ and $S$ in units of Planck mass.

The classical kinetic Lagrangian for the supergravity-matter-dilaton supermultiplet 
system is [1]

$$
\mathcal{L}_{0}=-3 \kappa^{-2} \int d^{4} \theta E
$$

with Kähler potential $K=K_{M}+K_{S}$ [1]. The kinetic Yang-Mills Lagrangian is given by [1]

$$
\mathcal{L}_{Y M}=\frac{1}{8} \int d^{4} \theta \frac{E}{R} S W^{\alpha} W_{\alpha}+\text { h.c. }
$$

where $W^{\alpha}$ denotes the Yang-Mills superfield fieldstrength. The gauge coupling constant $g$ is, at tree-level, given by the lowest component of $S, g^{-2}=\operatorname{Re} S \mid$. The complete kinetic Lagrangian,

$$
\mathcal{L}=\mathcal{L}_{0}+\mathcal{L}_{Y M}
$$

as well as the potential energy part of the Lagrangian and the partition function possess the following classical invariances.

1. Kähler invariance:

This follows from the transformation law of $E$, given in (2.4), under Kähler transformation (2.2).

2. Gauge invariance:

This follows from the fact that both $E$ and $R$ are functions of the Kähler potential $K$ which, in turn, is invariant under Yang-Mills transformations of the charged fields $\phi^{k}$ and $C_{d}$

$$
\begin{aligned}
e^{V} & \rightarrow e^{-i \bar{\Lambda}} e^{V} e^{i \Lambda} \\
\phi^{k} & \rightarrow e^{-i \Lambda} \phi^{k} \\
C_{d} & \rightarrow e^{-i \Lambda} C_{d}
\end{aligned}
$$

where

$$
\overline{\mathcal{D}}^{\dot{\alpha}} \Lambda=0
$$

and where $V$ denotes the Yang-Mills prepotential vector superfield. The square of the Yang-Mills superfield fieldstrength, $W^{\alpha} W_{\alpha}$, is by construction invariant under YangMills transformations. 
3. $\sigma$-model coordinate invariance and $S L(2, \mathcal{R})$ isometry invariance:

$\sigma$-model coordinate invariance of the partition function follows from the fact that, under these transformations, the Lagrangian and action, though not in general invariant, behave as scalars. When integrated over $\int \ldots\left[d \phi^{i}\right]\left[d \bar{\phi}^{\bar{j}}\right] \sqrt{\operatorname{det} g_{i \bar{j}}}$ in the path integral this assures that the partition function is invariant under $\sigma$-model coordinate transformations. The action of the $S L(2, \mathcal{R})$ isometry group, a subset of $\sigma$-model coordinate transformations, on a modulus $T^{I}$ can be represented by [15]

$$
T^{I} \rightarrow \frac{a T^{I}-i b}{i c T^{I}+d}
$$

where

$$
a d-b c=1, \quad a, b, c, d \in \mathcal{R}
$$

If the transformations (3.9) are accompanied by [15]

$$
\begin{aligned}
\phi^{i} & \rightarrow\left(\prod_{I}\left(i c T^{I}+d\right)^{-q_{i}^{I}}\right) \phi^{i}=e^{-\sum_{I} q_{i}^{I} F^{I}} \phi^{i} \\
C_{d} & \rightarrow\left(\prod_{I}\left(i c T^{I}+d\right)^{-q_{d}^{I}}\right) C_{d}=e^{-\sum_{I} q_{d}^{I} F^{I}} C_{d} \\
S & \rightarrow S
\end{aligned}
$$

where

$$
F^{I}=\ln \left(i c T^{I}+d\right)
$$

then $K$ transforms as

$$
\kappa^{2} K \rightarrow \kappa^{2} K+F(T)+\bar{F}(\bar{T})
$$

with

$$
F(T)=\sum_{I} F^{I}
$$

and, hence, the superdeterminant $E$ is invariant. It follows that the complete treelevel kinetic Lagrangian (3.6) is invariant under $S L(2, \mathcal{R})$. However, the potential energy part of the classical Lagrangian generically breaks $S L(2, \mathcal{R})$ invariance. The transformations (3.9) and (3.11) on the coordinates $\left(T^{I}, \phi^{i}, C_{d}\right)$ should be regarded as 
special continuous coordinate transformations on the $\left(T^{I}, \phi^{i}, C_{d}\right)$-manifold. Note that the modular transformations are the subset of these isometry transformations with $a, b, c, d \in \mathcal{Z}$. That is, the modular transformations correspond to the action of the $S L(2, \mathcal{Z})$ subgroup of $S L(2, \mathcal{R})$. The entire tree-level Lagrangian is $S L(2, \mathcal{Z})$ invariant, since orbifold potential energies respect this symmetry.

Invariances (1) - (3) of the tree-level theory of the supergravity-matter-dilaton supermultiplet system can, of course, also be displayed at component level. Component fields are defined according to standard notation [1]: $A^{i}, \chi_{\alpha}^{i}, \mathcal{F}^{i}$ for chiral multiplets (and similar notations for antichiral multiplets) and $\lambda_{\alpha}, v_{m}, \mathcal{D}$ for Yang-Mills multiplets. The covariant derivative of a generic Weyl fermion will, in a theory with invariances (1) - (3), then contain a connection for each of these symmetries.

1. The connection for gauging Kähler transformations (2.2) is given by the lowest component [1] of the $U_{K}(1)$ gauge connection superfield $A_{\alpha \dot{\alpha}}$ in (2.1)

$$
\begin{aligned}
A_{\alpha \dot{\alpha}} \mid & =a_{\alpha \dot{\alpha}}=\sigma_{\alpha \dot{\alpha}}^{m} a_{m} \\
a_{m} & =\frac{1}{4}\left(\partial_{j} K \mathcal{D}_{m} A^{j}-\partial_{\bar{j}} K \mathcal{D}_{m} \bar{A}^{\bar{j}}\right)+i \frac{1}{4} g_{i \bar{j}}\left(\chi^{i} \sigma^{m} \bar{\chi}^{\bar{j}}\right)
\end{aligned}
$$

2. The Yang-Mills connection $v_{\alpha \dot{\alpha}}=v_{\alpha \dot{\alpha}}^{(r)} T^{(r)}$ (where $T^{(r) a}{ }_{b}$ denote the generators of the Yang-Mills gauge group $G$ ) is contained in the Yang-Mills prepotential $V$

$$
\frac{1}{2}\left[\mathcal{D}_{\alpha}, \overline{\mathcal{D}}_{\dot{\alpha}}\right] V \mid=-2 v_{\alpha \dot{\alpha}}
$$

3. The connection $\Gamma^{i}{ }_{j k}$ which insures covariance under general coordinate transformations on a generic $\sigma$-model manifold of metric $g_{i \bar{j}}$ also insures covariance under isometries of the metric $g_{i \bar{j}}$, since these are a special subset of coordinate transformations. $\Gamma^{i}{ }_{j k}$ is given by $[2]$

$$
\Gamma^{i}{ }_{j k}=g^{i \bar{j}} \partial_{j} g_{k \bar{j}}
$$

For the Kähler potential (3.1) at hand, we find that, for the matter superfields $\phi^{i}$

$$
\Gamma_{J k}^{i}=\delta_{k}^{i} \partial_{J} \ln g_{i}
$$


where

$$
\ln \mathcal{G}_{i} \mid=\ln g_{i}
$$

Therefore, the complete covariant derivative for matter fermions $\chi_{\alpha}^{i a}$ reads [1]

$$
\begin{aligned}
\mathcal{D}_{m} \chi^{i} & =\partial_{m} \chi^{i}-\kappa^{2} a_{m} \chi^{i}+\left(\partial_{m} T^{J} \Gamma^{i}{ }_{J k}\right) \chi^{k} \\
& +i v_{m}^{(r)}\left(T^{(r)}-\frac{1}{2} D^{(r)}\right) \chi^{i}+\cdots
\end{aligned}
$$

where

$$
D^{(r)}=\kappa^{2} \frac{\partial K}{\partial A^{i a}} T_{b}^{(r) a} A^{i b}
$$

The dots stand for additional gravitational couplings such as the coupling to the spacetime Lorentz connection $\omega_{m}{ }^{\alpha}{ }_{\beta}$. Such additional couplings have been considered in a recent paper [3], and we will not include them here in our main discussion. They are, however, briefly commented on in Appendix A of this paper. Note that the connections $a_{m}$ and $\Gamma_{j k}^{i}$ are two totally distinct geometrical objects, as made explicit by the different powers of $\kappa^{2}$ multiplying them in in (3.20).

Of relevance for this paper are the couplings of the quantum currents to the connections discussed above, which will be taken to be external fields. We will consider quantum superfield fluctuations around a classical, $S L(2, \mathcal{R})$ preserving background $T^{I} \neq 0, \phi^{i}=0, C_{d}=0, S \neq 0$. This is consistent with the fact that higher order terms in $\phi^{i}$ and $C_{d}$ have been dropped in (3.1). Collecting all the relevant interaction terms in the component Lagrangian [1] we find the couplings of the quantum matter current $\bar{\chi}_{\dot{\alpha}}^{\bar{j} c} \chi_{\alpha}^{i a}$ to be

$$
\mathcal{L}_{i n t}=-\frac{i}{2} \bar{\chi}_{\dot{\alpha} a}^{\bar{j}}\left(\delta_{i \bar{j}} g_{i} \bar{\sigma}^{m \dot{\alpha} \alpha} X_{m}{ }^{a}{ }_{b i}\right) \chi_{\alpha}^{i b}+\mathcal{O}\left(\left(\chi^{i} \bar{\chi}^{\bar{j}}\right)^{2}\right)
$$

where

$$
\begin{aligned}
X_{m}{ }_{b i}^{a}= & -2 \kappa^{2} a_{m} \delta^{a}{ }_{b} \\
& +\left(\partial_{m} T^{I} \partial_{I} l_{I n g_{i}}-\partial_{m} \bar{T}^{\bar{I}} \partial_{\bar{I}} l n g_{i}+i \partial_{I} \partial_{\bar{J}} l n g_{i} \chi^{I} \sigma^{m} \bar{\chi}^{\bar{J}}\right) \delta^{a}{ }_{b} \\
& +2 i v_{m}^{(r)} T^{(r) a}{ }_{b}
\end{aligned}
$$

Note that the Killing potentials $D^{(r)}$ given in (3.21) do not appear in (3.23), since they vanish for the classical $S L(2, \mathcal{R})$ preserving background we have chosen. It will be 
useful to write $X_{m}{ }^{a}{ }_{b i}$ as the $\theta \bar{\theta}$-component of a prepotential superfield $Y$. It follows from (3.15), (3.16), and (3.19) that

$$
X_{m}{ }^{a}{ }_{b i}=\frac{i}{4} \bar{\sigma}_{m}^{\dot{\gamma} \gamma}\left[\mathcal{D}_{\gamma}, \overline{\mathcal{D}}_{\dot{\gamma}}\right] Y^{a}{ }_{b i} \mid
$$

where

$$
\begin{aligned}
Y_{b i}^{a} & =Z_{i} \delta^{a}{ }_{b}+V^{a}{ }_{b} \\
Z_{i} & =-\kappa^{2} \frac{1}{2} K+\ln \mathcal{G}_{i}
\end{aligned}
$$

Similarly, one finds that the couplings of the twisted quantum modulino currents $\bar{\chi}_{\dot{\alpha} d} \chi_{\alpha d}$ and the quantum gaugino currents $\bar{\lambda}_{\dot{\alpha}}^{b} \lambda_{\alpha}^{a}$ to the external gauge connections are again as in (3.24), where the prepotential superfields are now respectively given by

$$
\begin{aligned}
Y_{b d}^{a} & =Z_{d} \delta_{b}^{a}+V^{a}{ }_{b} \\
Y^{a}{ }_{b V} & =Z_{V} \delta^{a}{ }_{b}+V^{a}{ }_{b}
\end{aligned}
$$

where

$$
\begin{aligned}
Z_{d} & =-\kappa^{2} \frac{1}{2} K+\ln \mathcal{G}_{d} \\
Z_{V} & =\kappa^{2} \frac{1}{2} K
\end{aligned}
$$

Note that, due to the specific Yang-Mills kinetic term (3.5), there is an additional coupling of $\operatorname{Im} S \mid$ to the divergence of the gaugino current. $\operatorname{Im} S \mid$, however, does not transform under Kähler transformations. This is so, because superfield $S$ in (3.5) is inert under Kähler transformations. In fact, superfield $S$ coordinatizes the dilaton manifold and, hence, the coupling of $\operatorname{Im} S \mid$ to the divergence of the dilatino current in (3.5) should under no circumstances be reinterpreted as an additional coupling of the gaugino current to any of the connections under consideration. That is, this coupling is of no relevance for the discussion of $\sigma$-model anomalies and, hence, we will ignore it. In an analogous manner one finds that the couplings of the untwisted quantum modulino currents $\bar{\chi}_{\dot{\alpha}}^{I} \chi_{\alpha}^{I}$ to the external connections are contained in $X_{m I}$ which can be written as the $\theta \bar{\theta}$-component of a prepotential superfield as

$$
X_{m I}=\frac{i}{4} \bar{\sigma}_{m}^{\dot{\gamma} \gamma}\left[\mathcal{D}_{\gamma}, \overline{\mathcal{D}}_{\dot{\gamma}}\right] Z_{I}
$$


where

$$
Z_{I}=-\kappa^{2} \frac{1}{2} K+\ln \mathcal{G}_{I}
$$

and

$$
\mathcal{G}_{I}=\left.\frac{\partial^{2} K_{M}}{\partial T^{I} \partial \bar{T}^{\bar{I}}}\right|_{\langle\rangle}
$$

where $\left.\right|_{\langle\rangle}$means evaluation in the $S L(2, \mathcal{R})$ preserving background. Finally, the couplings of the twisted quantum dilatino current are as in (3.29), where the prepotential superfield is now given by

$$
Z_{S}=-\kappa^{2} \frac{1}{2} K+\ln \mathcal{G}_{S}
$$

where

$$
\mathcal{G}_{S}=\left.\frac{\partial^{2} K_{S}}{\partial S \partial \bar{S}}\right|_{\langle\rangle}
$$

Note that no mixed quantum currents, $\bar{\chi}_{\dot{\alpha}}^{i} \chi_{\alpha}^{I}$, between matter fermions and untwisted modulinos occur for the background chosen, and similarly for twisted modulinos.

\section{Kähler and $\sigma$-model Coordinate Anomalies in the Effective Theory}

In this section, we begin by evaluating non-local one-loop corrections associated with triangle graphs in the theory of the $E_{8} \otimes E_{6} \otimes U(1)^{2}$ orbifolds discussed in Section 3. We will work to lowest order in prepotentials $Z$ and $V$. These non-local terms will, in general, be anomalous under the symmetry transformations discussed in the previous section. Some of these terms can be straightforwardly generalized to the case of $(2,2)$ orbifolds with larger gauge group than $U(1)^{2}$, such as $Z_{3}$. As an example, the non-local term containing the Kähler prepotential $K$ only, can easily be generalized to the off-diagonal moduli case. For concreteness, we will, in Appendix A, discuss the pure Kähler anomaly for the $Z_{3}$ case, which we will find to be non-vanishing. This is the term generated by the triangle graph in which all three vertices couple to $K$.

We begin by considering matter fields $\phi^{k}$ and the associated prepotential superfield $Y_{k}$ given by (3.25). For the classical background chosen, the external Kähler prepotential $K$ in (3.25) is given by

$$
\kappa^{2} K=-\sum_{I} \ln \left(T^{I}+\bar{T}^{I}\right)-\ln (S+\bar{S})
$$


The transformation law of the prepotential superfied $Y_{k}$ can then be found from (2.2), (3.7) and (3.11), and reads, to linearized order,

$$
\begin{aligned}
\delta Y_{k} & =\delta Z_{k}+\delta V \\
\delta Z_{k} & =i \Lambda_{k}-i \bar{\Lambda}_{k} \\
\delta V & =i \Lambda-i \bar{\Lambda}
\end{aligned}
$$

where

$$
i \Lambda_{k}=-\frac{1}{2} \kappa^{2} F(T, S)+\sum_{I}\left(-\frac{1}{2}+q_{k}^{I}\right) F^{I}
$$

and

$$
\bar{D}^{\dot{\alpha}} \Lambda^{k}=\bar{D}^{\dot{\alpha}} \Lambda=0
$$

The classical Lagrangian (3.6) is invariant under gauge transformations (4.2). It is of interest to ask whether or not the quantum corrected effective action is invariant under (4.2) as well. As always in four space-time dimensions, one-loop non-local anomalous terms in the effective Lagrangian are associated with three-point triangle graphs [17]. The relevant supergraph is shown in Figure 1. In this graph, matter superfields $\phi^{k}$ run around the triangle loop and prepotential superfield $Y_{k}$ couples to each of the three matter field vertices, as discussed in Section 3. A similar graph has been considered in [18] in the context of supersymmetric, non-abelian chiral anomalies. There [18] it is the Yang-Mills prepotential superfield $V$ which couples to each of the three matter field vertices. The graph in [18] contributes a complicated non-local term to the effective action that has not been computed in [18]. Its variation under a Yang-Mills transformation, $\delta e^{V}$, however, has been computed in [18] in a field theoretical way as an infinite power series in $\left(e^{V}-1\right)$ using a Pauli-Villars regularization scheme. This result can be easily transcribed to our case, since the graph in Figure 1 is similar to the one discussed in [18], as mentioned. One first expands the result in [18] to lowest order in prepotential $V$, and subsequently replaces $V$ and its variation $\delta V=i \Lambda-i \bar{\Lambda}$ in [18] by $Y_{k}$ and its variation $\delta Y_{k}$ (as given by (4.2)). If we further take into account that the orbifold models are, by construction [4], anomaly free under Yang-Mills transformations, then the relevant transformation of prepotential superfield $Y_{k}$ is given by the parameters in (4.3) only. Thus, we arrive at the following finite and anomalous 
expression for the variation of the effective action $\Gamma$ under (4.3). It is

$$
\delta \Gamma_{\text {matter }}=\sum_{k}\left(i \Lambda_{k} \circ G-i \bar{\Lambda}_{k} \circ \bar{G}\right)
$$

where

$$
\begin{aligned}
i \Lambda_{k} \circ G & =\frac{1}{(4 \pi)^{2}} \frac{1}{(48)} \int d^{4} \theta i \Lambda_{k}\left[n_{k}^{E_{6}}\left(\bar{D}_{\dot{\alpha}} D^{\alpha} Z_{k}\right)\left(\bar{D}^{\dot{\alpha}} D_{\alpha} Z_{k}\right)\right. \\
& \left.+\operatorname{Tr}_{k}\left(3\left(\bar{D}_{\dot{\alpha}} D^{\alpha} V^{a}{ }_{c}\right)\left(\bar{D}^{\dot{\alpha}} D_{\alpha} V^{c}{ }_{b}\right)+3\left(\bar{D}_{\dot{\alpha}} D^{\alpha} V^{a}{ }_{b}\right)\left(\bar{D}^{\dot{\alpha}} D_{\alpha} Z_{k}\right)\right)\right]
\end{aligned}
$$

where $n_{k}^{E_{6}}=\operatorname{Tr}_{k}^{E_{6}} \delta^{a}{ }_{b}$. The relative factor of 3 is due to Bose symmetrization in the $Z Z Z$-channel. We would like to rewrite expression (4.6) in terms of superfield fieldstrengths defined as follows. The Yang-Mills superfield fieldstrength is, to lowest order in prepotentials, given by

$$
W_{\alpha}=-\frac{1}{8} \bar{D}^{2} D_{\alpha} V
$$

We defined another superfield fieldstrength with $Z_{k}$ as its prepotential field

$$
W_{\alpha k}=-\frac{1}{8} \bar{D}^{2} D_{\alpha} Z_{k}=-\frac{1}{2} X_{\alpha}-\frac{1}{8} \bar{D}^{2} D_{\alpha} \ln \mathcal{G}_{k}
$$

where $X_{\alpha}$ is given by (2.7). It is instructive as well as usefull to display the component fieldstrengths contained in $X_{\alpha}$ and $\bar{D}^{2} D_{\alpha} \ln \mathcal{G}_{k}$. These component fieldstrengths are contained in the $\theta$-component of $X_{\alpha}$ and $\bar{D}^{2} D_{\alpha} \ln \mathcal{G}_{k}$, respectively

$$
\begin{aligned}
\left.X_{\alpha}\right|_{\theta_{\beta}} & =\delta_{\alpha}^{\beta} \kappa^{2} D^{K}(y)-\left(\sigma^{m} \sigma^{n}\right)_{\alpha}^{\beta} \kappa^{2} v_{m n}^{K}(y) \\
-\left.\frac{1}{8} \bar{D}^{2} D_{\alpha} \ln \mathcal{G}_{k}\right|_{\theta_{\beta}} & =\delta_{\alpha}^{\beta} D_{k}(y)-\left(\sigma^{m} \sigma^{n}\right)_{\alpha}^{\beta} v_{m n}^{k}(y)
\end{aligned}
$$

In the above expression, $v_{m n}^{K}$ denotes the fieldstrength of the Kähler connection $a_{m}$ given in (3.15), $v_{m n}^{K}=\left(\partial_{m} a_{n}-\partial_{n} a_{m}\right)$, and $v_{m n}^{k}$ contains the fieldstrength of the $\sigma$ model manifold connection (3.18) pulled back to space-time

$$
\begin{aligned}
v_{m n}^{k} & =-\frac{1}{2} R_{I \bar{J}}^{k}\left(\partial_{m} T^{I} \partial_{n} \bar{T}^{\bar{J}}-(m \leftrightarrow n)\right) \\
& +\frac{i}{4} \partial_{m}\left(\left(\chi^{I} \sigma_{n} \bar{\chi}^{\bar{J}}\right) R_{I \bar{J}}^{k}\right)-(m \leftrightarrow n)
\end{aligned}
$$

where

$$
R_{I \bar{J}}^{k}=\partial_{I} \partial_{\bar{J}} \ln \mathcal{G}_{k}
$$


The $D$-terms in (4.9) are given [1] by

$$
\begin{aligned}
D^{K} & =\left(-g_{I \bar{J}} \partial_{m} T^{I} \partial^{m} \bar{T}^{\bar{J}}-g_{S \bar{S}} \partial_{m} S \partial^{m} \bar{S}+g_{I \bar{J}} \mathcal{F}^{I} \mathcal{F}^{\bar{J}}+g_{S \bar{S}} \mathcal{F}^{S} \mathcal{F}^{\bar{S}}\right) \\
D_{k} & =\left(-R_{I \bar{J}}^{k} \partial_{m} T^{I} \partial^{m} \bar{T}^{\bar{J}}+R_{I \bar{J}}^{k} \mathcal{F}^{I} \mathcal{F}^{\bar{J}}\right)
\end{aligned}
$$

Expression (4.6) can then be rewritten as

$$
i \Lambda_{k} \circ G=-\frac{1}{3} \frac{1}{(4 \pi)^{2}} \int d^{2} \theta i \Lambda_{k}\left[n_{k}^{E_{6}} W_{k}^{\alpha} W_{\alpha k}+3 \operatorname{Tr}_{k} W^{\alpha} W_{\alpha}+3\left(\operatorname{Tr}_{k} W^{\alpha}\right) W_{\alpha k}\right]
$$

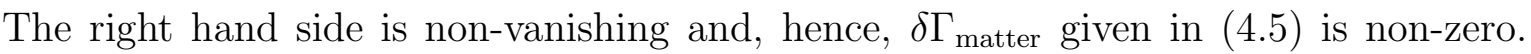
Therefore, the theory is anomalous under (4.3). Expression (4.13) can be obtained by variation of the following non-local term in the effective Lagrangian

$$
\begin{aligned}
\Gamma_{\text {matter }}= & \frac{1}{12} \frac{1}{(4 \pi)^{2}} \sum_{k} \int d^{4} \theta\left[n_{k}^{E_{6}} W_{k}^{\alpha} W_{\alpha k}\right. \\
& \left.+3 \operatorname{Tr}_{k}\left(W^{\alpha} W_{\alpha}\right)+3\left(\operatorname{Tr}_{k} W^{\alpha}\right) W_{\alpha k}\right] \frac{1}{\square} D^{2} Z_{k}+\text { h.c. }
\end{aligned}
$$

The anomalous contributions to the effective theory from the untwisted and twisted moduli superfields and the dilaton superfield running around a triangle loop can be calculated in a similar way. Each of these contributions can be readily obtained from (4.14) in the following way. As in (4.8), we first define superfield fieldstrengths $W_{\alpha I}$, $W_{\alpha d}$ and $W_{\alpha S}$ of the prepotential superfields $Z_{I}, Z_{d}$, and $Z_{S}$. Then, the contributions from the moduli and dilaton superfields are obtained from (4.14) by replacing $W_{\alpha k}$ and $Z_{k}$ with the corresponding $W$ and $Z$ for each of these fields. The contributions read

$$
\begin{aligned}
\Gamma_{\text {untwistedmoduli }}= & \frac{1}{12} \frac{1}{(4 \pi)^{2}} \sum_{I} \int d^{4} \theta W_{I}^{\alpha} W_{\alpha I} \frac{1}{\square} D^{2} Z_{I}+\text { h.c. } \\
\Gamma_{\text {twisted moduli }}= & \frac{1}{12} \frac{1}{(4 \pi)^{2}} \sum_{d} \int d^{4} \theta\left[W_{d}^{\alpha} W_{\alpha d}\right. \\
& \left.+3 \operatorname{Tr}_{d}\left(W^{\alpha} W_{\alpha}\right)+3\left(\operatorname{Tr}_{d} W^{\alpha}\right) W_{\alpha d}\right] \frac{1}{\square} D^{2} Z_{d}+\text { h.c. } \\
\Gamma_{\text {dilaton }}= & \frac{1}{12} \frac{1}{(4 \pi)^{2}} \int d^{4} \theta W_{S}^{\alpha} W_{\alpha S} \frac{1}{\square} D^{2} Z_{S}+\text { h.c. }
\end{aligned}
$$

The contribution from the gauginos has not been computed in [18]. However, it can be found in a similar way and reads

$$
\begin{aligned}
\Gamma_{\text {gaugino }} & =\frac{1}{12} \frac{1}{(4 \pi)^{2}} \sum_{H} \int d^{4} \theta\left[n_{V}^{H} W_{V}^{\alpha} W_{\alpha V}\right. \\
& \left.+3 \operatorname{Tr}_{V}^{H}\left(W^{\alpha} W_{\alpha}\right)+3\left(\operatorname{Tr}_{V}^{H} W^{\alpha}\right) W_{\alpha V}\right] \frac{1}{\square} D^{2} Z_{V}+\text { h.c. }
\end{aligned}
$$


where $H$ denotes the factor gauge groups $E_{8}, E_{6}$ and $U(1)^{2}$, and where $n_{V}^{H}=\operatorname{Tr}_{V}^{H} \delta^{a}{ }_{b}$. Thus, the sum of all the non-local contributions from the light fields to the effective action, as computed from triangle graphs, is, to lowest order in prepotentials, given by

$$
\Gamma_{\text {light }}=\Gamma_{V V Z}+\Gamma_{V Z Z}+\Gamma_{Z Z Z}
$$

where

$$
\begin{aligned}
\Gamma_{V V Z} & =\frac{1}{4} \frac{1}{(4 \pi)^{2}} \int d^{4} \theta\left[\sum_{k} \operatorname{Tr}_{k}\left(W^{\alpha} W_{\alpha}\right) \frac{1}{\square} D^{2} Z_{k}\right. \\
& \left.+\sum_{d} \operatorname{Tr}_{d}\left(W^{\alpha} W_{\alpha}\right) \frac{1}{\square} D^{2} Z_{d}+\operatorname{Tr}_{V}\left(W^{\alpha} W_{\alpha}\right) \frac{1}{\square} D^{2} Z_{V}\right]+ \text { h.c. }
\end{aligned}
$$

and

$$
\begin{aligned}
\Gamma_{V Z Z} & =\frac{1}{4} \frac{1}{(4 \pi)^{2}} \sum_{H} \int d^{4} \theta\left[\sum_{k}\left(\operatorname{Tr}_{k}^{H} W^{\alpha}\right) W_{\alpha k} \frac{1}{\square} D^{2} Z_{k}\right. \\
& \left.+\sum_{d}\left(\operatorname{Tr}_{d}^{H} W^{\alpha}\right) W_{\alpha d} \frac{1}{\square} D^{2} Z_{d}+\left(\operatorname{Tr}_{V}^{H} W^{\alpha}\right) W_{\alpha V} \frac{1}{\square} D^{2} Z_{V}\right]+ \text { h.c. } \\
\Gamma_{Z Z Z} & =\frac{1}{12} \frac{1}{(4 \pi)^{2}} \int d^{4} \theta\left[\sum_{k} n_{k}^{E_{6}} W_{k}^{\alpha} W_{\alpha k} \frac{1}{\square} D^{2} Z_{k}\right. \\
& +\sum_{I} W_{I}^{\alpha} W_{\alpha I} \frac{1}{\square} D^{2} Z_{I}+\sum_{d} W_{d}^{\alpha} W_{\alpha d} \frac{1}{\square} D^{2} Z_{d} \\
& \left.+\sum_{H} n_{V}^{H} W_{V}^{\alpha} W_{\alpha V} \frac{1}{\square} D^{2} Z_{V}+W_{S}^{\alpha} W_{\alpha S} \frac{1}{\square} D^{2} Z_{S}\right]+ \text { h.c. }
\end{aligned}
$$

$\Gamma_{V V Z}$ contains all graphs of the form $v v(a+\Gamma)$ and, hence, it contains the subset of graphs $v v(a+\Gamma)$ previously discussed in $[3,7,8,9,10]$ and given by (and generalized to the off-diagonal moduli case)

$$
\begin{aligned}
\Gamma_{V V Z}^{s u b} & =\frac{1}{4} \frac{1}{(4 \pi)^{2}} \sum_{H} \int d^{4} \theta\left(W_{H}^{(r) \alpha} W_{H(r) \alpha}\right) \frac{1}{\square} D^{2}\left\{\left(\frac{1}{2} \kappa^{2}\left(c_{V}^{H}-\sum_{R} c_{R}^{H}\right)\right) K\right. \\
& \left.+\sum_{R} c_{R}^{H} \ln \operatorname{det} \mathcal{G}_{R}\right\}+ \text { h.c. }
\end{aligned}
$$

where $R$ is any representation of charged chiral superfields, $\operatorname{Tr}_{R, V}^{H} T^{(r)} T^{(s)}=c_{R, V}^{H} \delta^{r s}$, and where $\mathcal{G}_{R}$ is that portion of the Kähler metric restricted to chiral superfields with representation $R$ of factor group $H$. It is this part of the effective action that is relevant to the computation of non-harmonic gauge couplings. The contributions $\Gamma_{V Z Z}$ 
and $\Gamma_{Z Z Z}$ correspond to all graphs of the form $v(a+\Gamma)^{2}$ and $(a+\Gamma)^{3}$, respectively. These terms have not previously been computed and are one of the main results of this paper. Note that they include a pure Kähler graph, aaa, a pure $\sigma$-model coordinate graph, ГГГ, as well as new mixed graphs such as $v a \Gamma$ and $a \Gamma^{2}$. Also note that mixed graphs such as $v a \Gamma$ and vaa contribute terms which are proportional to the trace of one single Yang-Mills generator, $\operatorname{Tr} T^{(r)}$, which need not vanish for the Yang-Mills gauge group under consideration, namely $E_{8} \otimes E_{6} \otimes U(1)^{2}$. These graphs render the theory anomalous under gauge transformations (4.2) and, hence, are just as dangerous, and just as interesting as the graphs contributing to $\Gamma_{V V Z}$. It is tedious, and not very enlightening, to rewrite all of $\Gamma_{V Z Z}$ and $\Gamma_{Z Z Z}$ in terms of $K, \ln \operatorname{det} \mathcal{G}_{I \bar{J}}$ and $\ln \operatorname{det} \mathcal{G}_{R}$. However, it is instructive to rewrite the terms in $\Gamma_{Z Z Z}$, which are proportional to the square of the Kähler fieldstrength $X_{\alpha}$ defined in (2.7), in this manner. This fieldstrength is contained in $W_{k}, W_{I}, W_{d}, W_{V}$ and $W_{S}$. The $X^{\alpha} X_{\alpha}$ terms in $\Gamma_{Z Z Z}$ (which we generalize to the off-diagonal moduli case) can be rewritten as

$$
\begin{aligned}
\Gamma_{X^{2}} & =\frac{1}{48} \frac{1}{(4 \pi)^{2}} \int d^{4} \theta X^{\alpha} X_{\alpha} \frac{1}{\square} D^{2}\left[\frac { 1 } { 2 } \kappa ^ { 2 } K \left(-\sum_{k}\left(\prod_{H} n_{k}^{H}\right)-\sum_{d}\left(\prod_{H} n_{d}^{H}\right)-n_{I}-1\right.\right. \\
& \left.\left.+\sum_{H} n_{V}^{H}\right)+\ln \operatorname{det} \mathcal{G}_{I \bar{J}}+\ln \mathcal{G}_{S}+\sum_{\text {sec }}\left(\prod_{H} n_{\text {sec }}^{H}\right) \ln \operatorname{det} \mathcal{G}_{\text {sec }}\right]+ \text { h.c. }
\end{aligned}
$$

where $n_{k, d}^{H}=\operatorname{Tr}_{k, d}^{H} \delta_{b}^{a}, n_{I}$ denotes the number of untwisted moduli, $\sum_{s e c}$ implies a sum over all sectors of matter and twisted moduli, $\mathcal{G}_{\text {sec }}$ is that portion of the Kähler metric restricted to the sector $s e c$, and where $\left(\prod_{H} n_{\text {sec }}^{H}\right)$ counts the Yang-Mills degrees of freedom in each of the sectors.

Let us discuss the anomalous behaviour of the various parts of $\Gamma_{\text {light }}$ under Kähler and $\sigma$-model coordinate transformations. To begin with, note that $K, \ln \operatorname{det} \mathcal{G}_{I \bar{J}}$ and ln $\operatorname{det} \mathcal{G}_{R}$, which are contained in the various superfields $Z$, are very different geometrical objects, as made explicit by the different powers of $\kappa^{2}$ multiplying them. As such, they will in general transform very differently under Kähler transformations and under general coordinate transformations on the moduli and matter manifold. Under Kähler transformations, only $K$ transforms whereas $\ln \operatorname{det} \mathcal{G}_{I \bar{J}}$ and $\ln \operatorname{det} \mathcal{G}_{R}$ are inert. Under general coordinate transformations, and, hence, under the subset of isometries, on the 
submanifold of the untwisted moduli, $K$ and $\ln \operatorname{det} \mathcal{G}_{R}$ transform as scalar functions

$$
\begin{aligned}
K^{\prime}\left(T^{\prime}, \bar{T}^{\prime}, S, \bar{S}\right) & =K(T, \bar{T}, S, \bar{S}) \\
\ln \operatorname{det} \mathcal{G}_{i \bar{j}}^{\prime}\left(T^{\prime}, \bar{T}^{\prime}\right) & =\ln \operatorname{det} \mathcal{G}_{i \bar{j}}(T, \bar{T})
\end{aligned}
$$

whereas $\ln \operatorname{det} \mathcal{G}_{I \bar{J}}$ transforms as the $\log$ of a density

$$
\ln \operatorname{det} \mathcal{G}_{I^{\prime} \bar{J}^{\prime}}^{\prime}\left(T^{\prime}, \bar{T}^{\prime}\right)=\ln \operatorname{det} \mathcal{G}_{I \bar{J}}(T, \bar{T})+\ln \operatorname{det} \Lambda_{I^{\prime}}{ }^{I}+\ln \operatorname{det} \bar{\Lambda}_{\bar{J}^{\prime}}{ }^{\bar{J}}
$$

where

$$
\Lambda_{I^{\prime}}^{I}=\frac{\partial T^{I}}{\partial T^{I^{\prime}}}
$$

Similarly, under coordinate transformations on the submanifold of matter fields with metric $\mathcal{G}_{R}$, the quantities $K$ and $\ln \operatorname{det} \mathcal{G}_{I \bar{J}}$ transform as scalar functions whereas $\ln \operatorname{det} \mathcal{G}_{R}$ transforms as the $\log$ of a density. First, let us apply these results to compute the variation of the various parts of $\Gamma_{\text {light }}$ under general Kähler transformations

$$
K \rightarrow K+F(T, S)+\bar{F}(\bar{T}, \bar{S})
$$

As an example, it is easy to see from (4.20) that under Kähler transformations (4.25) $\Gamma_{V V Z}^{\text {sub }}$ transforms as

$$
\delta_{K} \Gamma_{V V Z}^{s u b}=-\frac{1}{2} \frac{1}{(4 \pi)^{2}} \sum_{H} \int d^{2} \theta \kappa^{2} F(T, S)\left(W_{H}^{(r) \alpha} W_{H(r) \alpha}\right)\left(c_{V}^{H}-\sum_{R} c_{R}^{H}\right)+\text { h.c. }
$$

The right hand side of this expression is non-zero and, hence, $\Gamma_{V V Z}$ is Kähler anomalous. In a similar way, one can show that $\Gamma_{V Z Z}$ and $\Gamma_{Z Z Z}$ are anomalous as well under Kähler transformations. For example, it follows from (4.21) that $\Gamma_{X^{2}}$ transforms as

$$
\begin{aligned}
\delta_{K} \Gamma_{X^{2}} & =\frac{1}{24} \frac{1}{(4 \pi)^{2}} \int d^{2} \theta \kappa^{2} F(T, S) X^{\alpha} X_{\alpha}\left[\sum_{k}\left(\prod_{H} n_{k}^{H}\right)+\sum_{d}\left(\prod_{H} n_{d}^{H}\right)+n_{I}\right. \\
& \left.+1-\sum_{H} n_{V}^{H}\right]+ \text { h.c. }
\end{aligned}
$$

Again, the right hand side of this expression doesn't vanish and, hence, $\Gamma_{X^{2}}$ is Kähler anomalous. Note that $\delta_{K} \Gamma_{X^{2}}$ arises from a triangle graph in which all three vertices couple to $K$ only. Thus, it corresponds to a pure Kähler anomaly. Now let us consider the behaviour of the various parts of $\Gamma_{\text {light }}$ under $\sigma$-model coordinate transformations. 
It follows from (4.23) that $\Gamma_{X^{2}}$, given in (4.21), does not transform as a scalar under $\sigma$-model coordinate transformations on the submanifold of the untwisted moduli. Similarly, $\Gamma_{V V Z}^{s u b}$ given in (4.20) does not transform as a scalar under $\sigma$-model coordinate transformations on the submanifold of the matter fields. That is, the one-loop effective action $\Gamma_{\text {light }}$ does not transform as a scalar under $\sigma$-model coordinate transformations and, hence, the theory is anomalous under these transformations. Finally, let us consider the behaviour of the theory under a particular subset of $\sigma$-model coordinate transformations, namely under isometries (3.9) on the submanifold of the untwisted moduli. First consider $\Gamma_{V V Z}^{s u b}$ given in (4.20). We will choose the standard $(T, S)$-coordinate system in which $K$ takes the form (4.1). In this coordinate system, both $\ln \operatorname{det} \mathcal{G}_{I \bar{J}}$ and $\ln \operatorname{det} \mathcal{G}_{R}$ are proportional to $K-K_{S}$. $K$, however, transforms as in (3.13) under isometries (3.9), and, hence, $\Gamma_{V V Z}^{\text {sub }}$ transforms as

$$
\delta \Gamma_{V V Z}^{s u b}=-\frac{1}{2} \frac{1}{(4 \pi)^{2}} \sum_{H} \sum_{I} \int d^{2} \theta F^{I}\left(W_{H}^{(r) \alpha} W_{H(r) \alpha}\right)\left(c_{V}^{H}-\sum_{R} c_{R}^{H}\left(1-2 q_{R}^{I}\right)\right)+\text { h.c. }
$$

That is, $\Gamma_{V V Z}$ is anomalous under these isometries. Non-invariance of $\Gamma_{V V Z}^{s u b}$ under modular transformations, that is, isometries (3.9) for which $a, b, c, d \in \mathcal{Z}$, follows from this result and has been extensively discussed $[3,8,9]$ in the context of non-harmonic gauge couplings in string theory. In a similar way, one can see that the new contributions $\Gamma_{V Z Z}$ and $\Gamma_{Z Z Z}$ are anomalous under $\sigma$-model isometries. For example, it follows from (4.21) that $\Gamma_{X^{2}}$ transforms as

$$
\begin{aligned}
\delta \Gamma_{X^{2}} & =-\frac{1}{24} \frac{1}{(4 \pi)^{2}} \sum_{I} \int d^{2} \theta F^{I} X^{\alpha} X_{\alpha}\left[-\sum_{k}\left(\prod_{H} n_{k}^{H}\right)-\sum_{d}\left(\prod_{H} n_{d}^{H}\right)-n_{I}-1\right. \\
& \left.+\sum_{H} n_{V}^{H}+4+2 \sum_{\text {sec }}\left(\prod_{H} n_{\text {sec }}^{H}\right) q_{\text {sec }}^{I}\right]+h . c .
\end{aligned}
$$

and, hence, is non-invariant under isometries (3.9) (as well as under the subset of modular transformations).

We would like to point out again that, in the entire discussion given above, we have not included matter fields which are singlets under the $E_{6}$, since the structure of their Kähler potentials is unknown. They do, however, in general contribute to $\Gamma_{\text {light }}$ as well. Hence, one might ask whether their inclusion results in a cancellation of the various anomalous parts in $\Gamma_{\text {light }}$. We show, in Appendix A, that this does not happen. For concreteness, we consider in Appendix $\mathrm{A}$ the case of the $Z_{3}$ orbifold, for which 
the complete Kähler potential is known, and we show that $\Gamma_{X^{2}}$, and, hence, $\Gamma_{\text {light }}$, is non-vanishing.

Finally, it should also be pointed out that in the entire discussion given above, we have ignored the fact that the gravitino couples to the $U_{K}(1)$ connection as well. That is, we have avoided the issue of the quantization of supergravity fields. One might ask, however, whether the inclusion of the contribution of the gravitino field in (4.17) could result in a cancellation of the pure Kähler anomaly. From the appropriate indextheorem [19] for the spin $\frac{3}{2}$ operator of a Rarita-Schwinger spinor $\psi_{m}^{\alpha}$, it follows that the non-local contribution of the gravitino to $\Gamma_{X^{2}}$ is three times as big as the contribution of a single spin $\frac{1}{2}$ fermion with prepotential superfield $Z$ given by $Z=\frac{\kappa^{2}}{2} K$. Hence, the gravitino field contributes a factor -3 to the anomaly coefficient in (4.27). That is, the pure Kähler anomaly remains non-vanishing.

\section{Cancellation of Kähler and $\sigma$-model Coordinate Anomalies}

In this section we will propose a cancellation mechanism for the Kähler and $\sigma$ model coordinate anomalies in the effective theory of light fields, as computed in the previous section. We will proceed as follows. We will demand the theory to be Kähler invariant as well as invariant under $\sigma$-model coordinate transformations. To achieve this, we will have to postulate the existence of two types of counterterms, one for each type of symmetry transformation. These counterterms, when added to the effective Lagrangian of the light fields, will have to restore both types of symmetries. The presence of these counterterms will be interpreted as resulting from integrating out massive fields, such as Kaluza-Klein excitations and winding modes. We will be able to determine the moduli dependence of the two types of counterterms in the standard $(T, S)$-coordinate system from the requirement of modular invariance of the effective theory. Finally, we introduce an extension of the $\sigma$-model Green-Schwarz mechanism which will enable us to remove the universal part of $\Gamma_{X^{2}}$, as well as the universal part of the $\Gamma_{V V Z}$ term [10] discussed in the context of non-holomorphic gauge couplings in string theory. As in the previous sections, we will focus on the $E_{8} \otimes E_{6} \otimes U(1)^{2}$ orbifolds. We conjecture, however, that for other $(2,2)$ symmetric orbifolds, such as $Z_{3}$, all the steps carried out in our analysis remain valid. For concreteness, some explicit 
results are given in Appendix $\mathrm{A}$ for the case of the $Z_{3}$ orbifold.

We begin by demanding Kähler invariance of the effective theory of $(2,2)$ symmetric orbifolds. As pointed out in the last section, the effective Lagrangian $\Gamma_{\text {light }}$ is not invariant under Kähler transformations (4.25). Hence, a first type of counterterm has to be introduced. One way of rendering the effective theory Kähler invariant is to conjecture that the integration over massive modes conspires in such a way as to effectively induce a shift of the Kähler potential (4.1) in $\Gamma_{\text {light }}$ (contained in the $Z$ 's) by an amount

$$
\kappa^{2} K \rightarrow \kappa^{2} K+\ln |W|^{2} \equiv \kappa^{2} G(T, \bar{T}, S, \bar{S})
$$

Here, $W(T, S)$ denotes a yet unspecified superpotential. We have adopted a minimalestic principle. That is to say, we assume each term proportional to $K$ in $(4.17)$ gets shifted by the same amount $\ln |W|^{2}$.

Next, we would like to demand invariance of the partition function of $(2,2)$ symmetric orbifolds under $\sigma$-model coordinate transformations. We will proceed in two steps. In the first step we will demand the effective Lagrangian to transform as a scalar under general $\sigma$-model coordinate transformations. As pointed out in the last section, the effective Lagrangian $\Gamma_{\text {light }}$, computed from the light fields, does not transform as a scalar function under general coordinate transformations on the moduli, matter and dilaton manifold. This is so, because the contributions proportional to $Z_{A}$ (where $A=k, I, d, S)$ contain $\ln \mathcal{G}_{A}$ terms. A term $\ln \mathcal{G}_{A}$, however, transforms as the $\log$ of a density under an $A$-type $\sigma$-model coordinate transformation. Hence, a second set of counterterms, denoted $P_{A}$, has to be introduced. One way of achieving the scalar behaviour under $A$-type $\sigma$-model coordinate transformations is to conjecture that the integration over heavy modes contributes in such a way as to effectively shift the $\ln \mathcal{G}_{A}$ prepotentials in $\Gamma_{\text {light }}$ by amounts

$$
\ln \mathcal{G}_{A} \rightarrow \ln \mathcal{G}_{A}-P_{A}
$$

where

$$
P_{A}=\ln \hat{\mathcal{G}}_{A}
$$

and

$$
\hat{\mathcal{G}}_{A}=\partial_{A} \partial_{\bar{A}} P
$$


$P$ denotes some yet unspecified scalar function of the moduli, matter and dilaton fields. Then, $P_{A}$ clearly transforms as the $\log$ of a density under $A$-type $\sigma$-model coordinate transformations and, hence, the combination (5.2) transforms as a scalar function under any $\sigma$-model coordinate transformation. Note that the combination $\ln \mathcal{G}_{A}-P_{A}$ is Kähler invariant by construction.

We now demand that the effective theory be invariant under the $S L(2, \mathcal{Z})$ modular transformations, to conform to the expectation that superstring amplitudes are modular invariant [11]. Therefore, we must demand that the scalars $\ln \mathcal{G}_{A}-P_{A}$ and $G(T, \bar{T}, S, \bar{S})$ be invariant under the modular transformations of the untwisted moduli. We will choose the standard $(T, S)$-coordinate system, in which the Kähler potential $K$ is given by (4.1). In particular, then, by demanding the scalars $\ln \mathcal{G}_{A}-P_{A}$ and $G(T, \bar{T}, S, \bar{S})$ to be separately invariant under modular transformations, we will be able to find the explicit form of some of the counterterms in the standard $(T, S)$-coordinate system. First, let us discuss $G(T, \bar{T}, S, \bar{S})$. If we make the ansatz

$$
W(T, S)=\Omega(S) W(T)
$$

then

$$
G(T, \bar{T}, S, \bar{S})=G(T, \bar{T})+G(S, \bar{S})
$$

where

$$
\begin{aligned}
G(T, \bar{T}) & =K_{M}(T, \bar{T})+\kappa^{-2} \ln |W(T)|^{2} \\
G(S, \bar{S}) & =K_{S}(S, \bar{S})+\kappa^{-2} \ln |\Omega(S)|^{2}
\end{aligned}
$$

Demanding the invariance of $G(T, \bar{T}, S, \bar{S})$ under modular transformations, the $S L(2, \mathcal{Z})$ subset of isometries (3.9), restricts $W(T)$ to be the modular function

$$
W(T)=\prod_{I} \eta^{-2}\left(i T^{I}\right)
$$

It follows that

$$
\kappa^{2} G(T, \bar{T})=-\sum_{I} \ln \left(T^{I}+\bar{T}^{I}\right)\left|\eta\left(i T^{I}\right)\right|^{4}
$$

The explicit form of $W(T)$, and, hence, of $G(T, \bar{T})$ agrees with recent discussions in the literature [9]. Note that $\Omega(S)$ remains unspecified. Next, let us discuss the 
counterterms $P_{A}, A=k, d$, for matter fields and twisted moduli. It follows from (3.2) that $\ln \mathcal{G}_{A}$ is given as

$$
\ln \mathcal{G}_{A}=\sum_{I}\left(-q_{A}^{I} \ln \left(T^{I}+\bar{T}^{I}\right)\right)
$$

Hence, the scalar $\ln \mathcal{G}_{A}-P_{A}$ is invariant under the modular transformations provided that

$$
\hat{\mathcal{G}}_{A}=\prod_{I}\left(\left|\eta\left(i T^{I}\right)\right|^{4}\right)^{q_{A}^{I}}
$$

The scalars $\ln \mathcal{G}_{A}-\ln \hat{\mathcal{G}}_{A}, A=k, d$, have the same form as the one-loop corrections to inverse gauge couplings in string theory computed in $[7,9]$. Similarly, the counterterm $\hat{\mathcal{G}}_{I}$ for untwisted moduli is determined to be

$$
\hat{\mathcal{G}}_{I}=\prod_{I}\left(\left|\eta\left(i T^{I}\right)\right|^{4}\right)^{2}
$$

Note that the counterterm $P_{S}$ remains unspecified. Although the counterterms (5.8), (5.11) and (5.12) restore invariance of the effective Lagrangian under $S L(2, \mathcal{Z})$ transformations, they do not restore the full $S L(2, \mathcal{R})$ isometry invariance of the tree-level kinetic Lagrangian (3.6). That is, the $S L(2, \mathcal{R})$ symmetry of the tree-level kinetic Lagrangian is broken down to $S L(2, \mathcal{Z})$ modular invariance at the one-loop level.

We have rendered the theory of $(2,2)$ symmetric orbifolds invariant under Kähler transformations and under general $\sigma$-model coordinate transformations by introducing two types of counterterms, one for each type of symmetry transformation. A priori, there is then no need for any additional mechanism for restoring the symmetries under consideration. However, it has been shown [10] in explicit calculations of string theory scattering amplitudes that there is yet another removal mechanism at work, namely a $\sigma$-model generalization [8] of the Green-Schwarz mechanism [12]. Hence, we now proceed to discuss extensions of the Green-Schwarz mechanism for any $(2,2)$ symmetric orbifold [8]. The Green-Schwarz term, denoted $\mathcal{L}_{G S}$, utilizes the linear multiplet representation $l$ for the dilaton supermultiplet [20]. The linear multiplet $l$ is defined to satisfy the following modified linearity condition in Kähler superspace

$$
\begin{aligned}
\left(\overline{\mathcal{D}}^{2}-8 R\right) l= & -8 \sum_{H} t_{H}\left(W_{H}^{(r) \alpha} W_{H \alpha}^{(r)}\right)+\frac{1}{6}(3 \sigma+4 \tau) X^{\alpha} X_{\alpha} \\
& -8 \tau\left(W^{\alpha \beta \gamma} W_{\alpha \beta \gamma}\right)
\end{aligned}
$$




$$
\begin{aligned}
\left(\mathcal{D}^{2}-8 R^{+}\right) l= & -8 \sum_{H} t_{H}\left(\bar{W}_{H \dot{\alpha}}^{(r)} \bar{W}_{H}^{(r) \dot{\alpha}}\right)+\frac{1}{6}(3 \sigma+4 \tau) \bar{X}_{\dot{\alpha}} \bar{X}^{\dot{\alpha}} \\
& -8 \tau\left(\bar{W}_{\dot{\alpha} \dot{\beta} \dot{\gamma}} W^{\dot{\alpha} \dot{\beta} \dot{\gamma}}\right)
\end{aligned}
$$

The $X^{\alpha} X_{\alpha}$ terms in (5.13) correspond to a Chern-Simons term for the Kähler symmetry and are a generalization of the $U_{R}(1)$ Chern-Simons terms introduced in [21]. Equation (5.13) describes the most general coupling of a linear multiplet to the square of gauge, Kähler and gravitational superfield fieldstrengths. That is, in a general supergravity theory the coefficients $t_{H}, \sigma$ and $\tau$ in (5.13) can be taken to have arbitrary values. In the case of the superstring inspired supergravity theories we are mainly concerned with, these coefficients have fixed values. For instance, one finds that, if the description of the dilaton supermultiplet in the chiral $S$ picture is to be equivalent to its description in the $l$ picture, each of the Yang-Mills coefficients $t_{H}$ has to be taken to satisfy

$$
t_{H}=\frac{1}{24}
$$

Then, it can be shown [20] that, upon duality transformation from the chiral superfield $S$ to $l$, the tree-level Lagrangian (3.6) in the chiral $S$ picture is equivalent to the following Lagrangian in the $l$ picture

$$
\mathcal{L}=-3 \kappa^{-2} \int d^{4} \theta \frac{2}{3} E
$$

where $E=E[\hat{K}]$ with $\hat{K}=K_{M}+\ln l$, and where $l$ satisfies (5.13) with coefficients $t_{H}$ given by (5.14). Equivalence of tree-level Lagrangians (3.6) and (5.15) also implies that the coefficients $\sigma$ and $\tau$ vanish. In the following, however, we will allow for nonvanishing $\sigma$ and $\tau$ and, hence, we will discuss extensions of the supergravity theories given by (3.6). That is, we will allow for additional couplings of the dilaton $S$ to the square of Kähler and gravitational superfield field strengths. In fact, by generalizing the duality transformation given in [20] it can be shown that the Kähler and gravitational contributions on the right hand side of (5.13) contribute the following additional terms to the tree-level Lagrangian (3.6) in the $S$-picture

$$
\mathcal{L}^{\prime}=-\frac{(3 \sigma+4 \tau)}{16} \int d^{4} \theta \frac{E}{R} S X^{\alpha} X_{\alpha}+3 \tau \int d^{4} \theta \frac{E}{R} S W^{\alpha \beta \gamma} W_{\alpha \beta \gamma}+\text { h.c. }
$$

We will determine the coefficients $\sigma$ and $\tau$ in the following way. We will conjecture that a part (perhaps all) of the universal part of the mixed $\sigma$-model gravitational 
anomalies, as discussed in [3] and in Appendix A, as well as a part (perhaps all) of the pure Kähler anomaly get removed by an extension of the Green-Schwarz mechanism. This will uniquely fix both coefficients $\sigma$ and $\tau$. Hence, removal of these universal parts in string theory requires the presence of the additional tree-level Kähler and gravitational couplings (5.16).

We proceed to discuss the $\sigma$-model generalization of the Green-Schwarz mechanism. We first expand (5.13) to lowest order in the prepotentials $V$ and $K$, and we find that

$$
\begin{aligned}
\bar{D}^{2} l & =-\frac{1}{3} \sum_{H}\left(W_{H}^{(r) \alpha} W_{H \alpha}^{(r)}\right)+\frac{1}{6}(3 \sigma+4 \tau) X^{\alpha} X_{\alpha} \\
D^{2} l & =-\frac{1}{3} \sum_{H}\left(\bar{W}_{H \dot{\alpha}}^{(r)} \bar{W}_{H}^{(r) \dot{\alpha}}\right)+\frac{1}{6}(3 \sigma+4 \tau) \bar{X}_{\dot{\alpha}} \bar{X}^{\dot{\alpha}}
\end{aligned}
$$

Note that, to lowest order in the prepotentials $V$ and $K$, the superfield $W_{\alpha \beta \gamma}$ doesn't contribute to the right hand side of equation (5.17). Also note that, on the right hand side of (5.17), there is no superfield fieldstrength's square associated with prepotentials ln $\mathcal{G}_{A}$. The Green-Schwarz term, which we will take to be of order $\hbar$, has the generic form

$$
\mathcal{L}_{G S}=\beta \int d^{4} \theta E l \Xi+\text { h.c. }
$$

where $\beta$ is real and where $\Xi$ is taken to be a scalar function of the untwisted moduli $T^{I}$, invariant under Kähler transformations and under modular transformations. Hence, the addition of the term $\mathcal{L}_{G S}$ to the Lagrangian of the effective theory (already supplemented with appropriate counterterms, as discussed above) will maintain both Kähler and modular invariances of the theory. The coefficient $\beta$ as well as the scalar function $\Xi$ in (5.18) can be determined from the knowledge (in any $(2,2)$ symmetric $Z_{N}$ orbifold) of the absence of dependence of the effective $E_{8}$ gauge coupling constant on the untwisted moduli [10]. We proceed as follows. We first solve (5.17) for $l$

$$
\begin{aligned}
l & =\frac{1}{16} \frac{D^{2}}{\square}\left\{-\frac{1}{3} \sum_{H}\left(W_{H}^{(r) \alpha} W_{H \alpha}^{(r)}\right)+\frac{1}{6}(3 \sigma+4 \tau) X^{\alpha} X_{\alpha}\right\} \\
& +\frac{1}{16} \frac{\bar{D}^{2}}{\square}\left\{-\frac{1}{3} \sum_{H}\left(\bar{W}_{H}^{(r)}{ }_{\dot{\alpha}} \bar{W}_{H}^{(r) \dot{\alpha}}\right)+\frac{1}{6}(3 \sigma+4 \tau) \bar{X}_{\dot{\alpha}} \bar{X}^{\dot{\alpha}}\right\}
\end{aligned}
$$

Then, inserting (5.19) into (5.18) yields the following non-local contribution $\Gamma_{G S}$ to the 
effective theory

$$
\begin{aligned}
\Gamma_{G S} & =-\frac{1}{24} \beta \sum_{H} \int d^{4} \theta\left(W_{H}^{(r) \alpha} W_{H \alpha}^{(r)}\right) \frac{1}{\square} D^{2} \Xi \\
& +\frac{1}{8} \beta \frac{(3 \sigma+4 \tau)}{6} \int d^{4} \theta X^{\alpha} X_{\alpha} \frac{1}{\square} D^{2} \Xi+h . c .
\end{aligned}
$$

The two terms in $\Gamma_{G S}$ stem from reducible tree graphs similar to the one shown in Figure 2. We will call them contributions of the universal type. We now compare $\Gamma_{G S}$ in (5.20) against $\Gamma_{V V Z}^{s u b}$ in (4.20). We supplement $\Gamma_{V V Z}^{s u b}$ with counterterms (5.1) and (5.4) and we denote the resulting expression by $\Gamma_{V V Z}^{\prime s u b}$. It is useful to rewrite $\Gamma_{V V Z}^{\prime s u b}$ in the following way

$$
\begin{aligned}
\Gamma_{V V Z}^{\prime s u b} & =\frac{1}{4} \frac{1}{(4 \pi)^{2}} \sum_{H} \int d^{4} \theta\left(W_{H}^{(r) \alpha} W_{H(r) \alpha}\right) \frac{1}{\square} D^{2}\left(\frac{1}{2} \kappa^{2} c_{V}^{E_{8}}\right) G \\
& +\frac{1}{4} \frac{1}{(4 \pi)^{2}} \sum_{H} \int d^{4} \theta\left(W_{H}^{(r) \alpha} W_{H(r) \alpha}\right) \frac{1}{\square} D^{2}\left\{\left(\frac{1}{2} \kappa^{2}\left(-c_{V}^{E_{8}}+c_{V}^{H}-\sum_{R} c_{R}^{H}\right)\right) G\right. \\
& \left.+\sum_{R} c_{R}^{H}\left(\ln \operatorname{det} \mathcal{G}_{R}-\ln \operatorname{det} \hat{\mathcal{G}}_{R}\right)\right\}+ \text { h.c. }
\end{aligned}
$$

The first sum on the right hand side of $\Gamma_{V V Z}^{\prime s u b}$ yields a moduli dependent contribution to the effective $E_{8}$ gauge coupling constant. It has been shown [10], however, that such a contribution is absent in the effective theory of any $(2,2)$ symmetric $Z_{N}$ orbifold. Hence, we will remove this contribution in the following way. By noting that the term $\kappa^{2} c_{V}^{E_{8}} G(T, \bar{T})$ in $\Gamma_{V V Z}^{\prime s u b}$ is of the universal type and by comparing $\Gamma_{V V Z}^{\prime s u b}$ against $\Gamma_{G S}$ in (5.20), it follows that the term $\kappa^{2} c_{V}^{E_{8}} G(T, \bar{T})$ can be removed from $\Gamma_{V V Z}^{\prime s u b}$ by choosing

$$
\Xi=\kappa^{2} G(T, \bar{T})
$$

and

$$
\beta=3 \frac{1}{(4 \pi)^{2}} c_{V}^{E_{8}}
$$

where, in the standard coordinate system, $G(T, \bar{T})$ is given by $(5.9)$. $\Gamma_{G S}$ now becomes

$$
\Gamma_{G S}^{\prime}=\frac{1}{8} \beta \frac{(3 \sigma+4 \tau)}{6} \int d^{4} \theta X^{\alpha} X_{\alpha} \frac{1}{\square} D^{2} \kappa^{2} G(T, \bar{T})+h . c .
$$

We now compare $\Gamma_{G S}^{\prime}$ against $\Gamma_{X^{2}}$ in (4.21). We supplement $\Gamma_{X^{2}}$ with counterterms (5.1) and (5.4) and we denote the resulting expression by $\Gamma_{X^{2}}^{\prime}$. We rewrite $\Gamma_{X^{2}}^{\prime}$ as 
follows

$$
\begin{aligned}
\Gamma_{X^{2}}^{\prime} & =\frac{\alpha}{96} \frac{1}{(4 \pi)^{2}} \int d^{4} \theta X^{\alpha} X_{\alpha} \frac{1}{\square} D^{2} \kappa^{2} G \\
& +\frac{1}{48} \frac{1}{(4 \pi)^{2}} \int d^{4} \theta X^{\alpha} X_{\alpha} \frac{1}{\square} D^{2}\left[\frac { 1 } { 2 } \kappa ^ { 2 } G \left(-\alpha-\sum_{k}\left(\prod_{H} n_{k}^{H}\right)-\sum_{d}\left(\prod_{H} n_{d}^{H}\right)\right.\right. \\
& \left.-n_{I}-1+\sum_{H} n_{V}^{H}\right)+\ln \operatorname{det} \mathcal{G}_{I \bar{J}}-\ln \operatorname{det} \hat{\mathcal{G}}_{I \bar{J}}+\ln \mathcal{G}_{S}-\ln \hat{\mathcal{G}}_{S} \\
& \left.+\sum_{\text {sec }}\left(\prod_{H} n_{\text {sec }}^{H}\right)\left(\ln \operatorname{det} \mathcal{G}_{\text {sec }}-\ln \operatorname{det} \hat{\mathcal{G}}_{\text {sec }}\right)\right]+ \text { h.c. }
\end{aligned}
$$

where $\alpha$ denotes an yet unspecified constant. Note that $\Gamma_{X^{2}}^{\prime}$ contains terms which are of the universal type (5.24). These terms stem from the pure Kähler graph aaa. We now conjecture that a certain amount of the universal type gets removed by the Green-Schwarz mechanism as well. We will denote the amount by $\alpha$. The actual value of $\alpha$ has yet to be determined by an explicit calculation of the appropriate three-point string scattering amplitude. Here, we assume that $\alpha$ is non-vanishing. By comparing $\Gamma_{X^{2}}^{\prime}$ against $\Gamma_{G S}^{\prime}$, it follows that the first term in $\Gamma_{X^{2}}^{\prime}$ can be removed by choosing

$$
3 \sigma+4 \tau=-\alpha \frac{1}{6 c_{V}^{E_{8}}}
$$

We would like to point out again, that we have omitted the contributions from matter singlets to $\Gamma_{X^{2}}^{\prime}$, since the structure of their Kähler potentials is unknown. For concreteness, we will, in Appendix A, discuss the Green-Schwarz mechanism for the case of the $Z_{3}$ orbifold, for which the complete Kähler potential is known. Let us also emphasize again that, whether or not the removal (and how much) of a universal piece of $\Gamma_{X^{2}}^{\prime}$ by the Green-Schwarz mechanism takes place in the effective theory, has yet to be checked by an explicit calculation of the appropriate three-point scattering amplitude.

We have, so far, only determined the linear combination $3 \sigma+4 \tau$ of coefficients $\tau$ and $\sigma$. We will now determine $\tau$ and $\sigma$ individually, as follows. The coefficient $\tau$ can be determined by conjecturing that an amount $\gamma$ of the anomalous mixed $\sigma$ model gravitational 1-loop contributions to $\Gamma_{\text {light }}$ is removed by the Green-Schwarz mechanism as well. Such contributions arise from triangle graphs $\omega \omega a$ and $\omega \omega \Gamma$, where $\omega$ denotes the space-time Lorentz-connection. We have not discussed such graphs in this paper; they have, however, been discussed in [3]. In Appendix A we will compute 
these mixed gravitational contributions for the case of the $Z_{3}$ orbifold, as an example. We also include the contribution from the gravitino [22], which had been missing in [3]. Similarly to the $Z_{3}$ case, the contributions of the universal type in $U(1)^{2}$ orbifolds arise in the graph $\omega \omega a$. These contributions to $\Gamma_{\text {light }}$ are proportional to $K$ and have the form

$$
\Gamma_{\text {gravity }}^{\text {univ }}=\frac{1}{12} \frac{1}{(4 \pi)^{2}} \int d^{4} \theta W^{\alpha \beta \gamma} W_{\alpha \beta \gamma} \frac{1}{\square} D^{2}(\gamma+(c-\gamma)) \kappa^{2} K+\text { h.c. }
$$

where the coefficient $c$ can be determined from the number of massless states in the orbifold spectrum, and where $\gamma$ denotes the amount of universal type we conjecture is removed by the Green-Schwarz mechanism. The removal of the amount $\gamma$ fixes the coefficient $\tau$ to be equal to

$$
\tau=\gamma \frac{1}{36 c_{V}^{E_{8}}}
$$

Then, the coefficient $\sigma$ is determined from (5.26) and (5.28) to be equal to

$$
\sigma=-\left(\frac{\gamma}{3}+\frac{\alpha}{2}\right) \frac{1}{9 c_{V}^{E_{8}}}
$$

The non-vanishing of the coefficients $\tau$ and $\sigma$ leads to the addition of the new contributions (5.16) to the tree-level Lagrangian (3.6) in the S-picture. For concretenes, we would like to display the bosonic component content of the term in (5.16) proportional to the square of the Kähler superfield fieldstrength $X^{2}$

$$
\mathcal{L}_{X^{2}}^{\prime}=-\frac{1}{8}(3 \sigma+4 \tau) \kappa^{4} S \mid\left(2 v_{m n}^{K} v^{K m n}+i \epsilon^{k l m n} v_{k l}^{K} v_{m n}^{K}+\left(D^{K}\right)^{2}\right)+\text { h.c. }
$$

where $S \mid$ denotes the lowest component of the dilaton superfield $S$, where $v_{m n}^{K}$ denotes the fieldstrength of the component Kähler connection $(3.15), v_{m n}^{K}=\partial_{m} a_{n}-\partial_{n} a_{m}$, and where $D^{K}$ is given in (4.12). Finally, let us point out again that, in the above discussion, we have not been able to fix the form of the counterterms $\Omega(S)$ and $P_{S}$.

\section{Conclusion}

We have calculated and discussed the complete set of one-loop triangle graphs involving the Yang-Mills gauge connection, the Kähler connection and the $\sigma$-model coordinate connection in the effective field theory of $(2,2)$ symmetric $Z_{N}$ orbifolds. We 
have shown that each of these graphs is anomalous under both Kähler transformations and $\sigma$-model coordinate transformations. We have demonstrated that, upon introducing certain counterterms, it is possible to preserve both Kähler and $\sigma$-model coordinate invariances of $Z_{N}$ orbifolds. We have been able to determine the moduli dependence of these counterterms by demanding $S L(2, \mathcal{Z})$ modular invariance of the effective theory of $Z_{N}$ orbifolds. The $S L(2, \mathcal{R})$ symmetry of the tree-level kinetic Lagrangian is broken by the counterterms down to $S L(2, \mathcal{Z})$ modular invariance. Additional explicit calculations of on-shell three-point correlation functions (involving the untwisted moduli and the dilaton) are necessary to determine the dilaton dependence of these counterterms. We have also discussed the possibility of removing the universal part of the contribution from the pure Kähler graph aaa by the Green-Schwarz mechanism. Whether or not such a removal takes place in the effective theory has yet to be checked by an explicit calculation of the appropriate composite three-point scattering amplitude. The relevant on-shell three-point correlation function consists of two Kähler gauge fields $a_{m}, a_{n}$ and one untwisted modulus $T$. We would like to emphasize the importance of such calculations and to urge people to do them.

\section{Acknowledgements}

We would like to thank M. Cvetič for many informative discussions. We are grateful to R. Grimm for many fruitful discussions on the geometry of Kähler superspace.

\section{Appendix A}

We give a detailed discussion of the anomalous mixed $\sigma$-model gravitational 1-loop contributions as well as of the $\Gamma_{X^{2}}$ contribution to $\Gamma_{\text {light }}$ for the case of the $Z_{3}$ orbifold. We begin with the anomalous mixed $\sigma$-model gravitational 1-loop contributions. Such contributions arise from triangle graphs $\omega \omega a$ and $\omega \omega \Gamma$, where $\omega$ denotes the space-time Lorentz connection. They are invariant under space-time Lorentz transformations, since, as it is well known [19], there are no pure space-time gravitational anomalies in four dimensions. The anomalous mixed $\sigma$-model gravitational 1-loop contributions 
were discussed in [3] and found to be equal to

$$
\Gamma_{\text {gravity }}=\frac{1}{12} \frac{1}{(4 \pi)^{2}} \int d^{4} \theta W^{\alpha \beta \gamma} W_{\alpha \beta \gamma} \frac{1}{\square} D^{2}\left(c \kappa^{2} K+N\right)+\text { h.c. }
$$

where

$$
c=720
$$

and

$$
\begin{aligned}
N & =\kappa^{2} K\left(-c-\sum_{k}\left(\prod_{H} n_{k}^{H}\right)-\sum_{d}\left(\prod_{H} n_{d}^{H}\right)-n_{I}-1+\sum_{H} n_{V}^{H}-21\right) \\
& +2 \ln \operatorname{det} \mathcal{G}_{I \bar{J}}+2 \ln \mathcal{G}_{S}+2 \sum_{\text {sec }}\left(\prod_{H} n_{\text {sec }}^{H}\right) \ln \operatorname{det} \mathcal{G}_{\text {sec }}
\end{aligned}
$$

The quantities on the right hand side of equation (A.3) have been defined in section 4 of this paper. In (A.3), we have included the contribution from the dilatino, -1 , as well as from the gravitino, -21 . The latter contribution was computed in [22] and can be readily obtained from the appropriate index-theorem [19] for the spin $\frac{3}{2}$ operator of a Rarita-Schwinger spinor. We would like to compute (A.3) for the $Z_{3}$ case and show that, in the standard $(T, S)$-coordinate system, the dependence of $N$ on the untwisted moduli cancels out. We begin by computing the coefficient of the $K$-term in (A.3). The gauge group $G$ of $Z_{3}, G=E_{8} \otimes E_{6} \otimes S U(3)$, contains $\sum_{H} n_{V}^{H}=334$ generators. Massless chiral superfields occur in both the untwisted and twisted sectors. In the untwisted sector, there are nine $(1,1)$ moduli superfields, denoted $T_{I \bar{J}}$ where $I, \bar{J}=1,2,3$, which transform as $(\mathbf{1}, \mathbf{1}, \mathbf{1})$ under $G$. In addition there are three matter superfields which transform as $(\mathbf{1}, \mathbf{2 7}, \mathbf{3})$ under $G$. In the twisted sector there are twenty seven matter superfields which transform as $(\mathbf{1}, \mathbf{2 7 , 1})$ under $G$ as well as eighty-one superfields which transform as $(\mathbf{1}, \mathbf{1}, \overline{\mathbf{3}})$ under $G$. Inserting this information into (A.3) yields

$$
-c-\sum_{k}\left(\prod_{H} n_{k}^{H}\right)-\sum_{d}\left(\prod_{H} n_{d}^{H}\right)-n_{I}-1+\sum_{H} n_{V}^{H}-21=-1632
$$

Next, we compute the terms proportional to $\ln \operatorname{det} \mathcal{G}$ in (A.3). This requires the knowledge of the modular weights $q_{\text {sec }}^{I}$ (see (3.2)). They are equal to 1 for the untwisted matter fields, $\frac{2}{3}$ for the twisted $(\mathbf{1}, \mathbf{2 7}, \mathbf{1})$-multiplets and 1 for the twisted $(\mathbf{1}, \mathbf{1}, \overline{\mathbf{3}})$ multiplets. Furthermore, it can be shown [3] that ln $\operatorname{det} \mathcal{G}_{\text {moduli }}=6 K_{M}$. Inserting this 
information into (A.3) and retaining only the dependence on the untwisted moduli yields

$$
2 \ln \operatorname{det} \mathcal{G}_{I \bar{J}}+2 \sum_{\text {sec }}\left(\prod_{H} n_{\text {sec }}^{H}\right) \ln \operatorname{det} \mathcal{G}_{\text {sec }}=1632 K_{M}
$$

Hence, the moduli dependent part of $N$ cancels out. Note that, when supplementing (A.1) with the counterterms introduced in the main part of this paper, the moduli dependence of these counterterms cancels out as well. Since the introduction of these counterterms renders $N$ a scalar function of the untwisted moduli, its moduli dependence cancels out in any coordinate system. It is then tempting to conjecture that the coefficient $c$ given in (A.2) represents the amount of the $K$-terms in (A.1), which is removed by the Green-Schwarz mechanism in the following way. The term proportional to $\left(W_{\alpha \beta \gamma}\right)^{2}$ in (5.13), when inserted into the Green-Schwarz Lagrangian (5.18), contributes the following non-local term $\Gamma_{G S}$ to the effective theory

$$
\Gamma_{G S}=-\beta \tau \int d^{4} \theta W^{\alpha \beta \gamma} W_{\alpha \beta \gamma} \frac{1}{\square} D^{2} \kappa^{2} G+\text { h.c. }
$$

By comparing (A.6) against (A.1) it follows that the amount $c$ can be removed when choosing

$$
\tau=\frac{720}{\beta} \frac{1}{12} \frac{1}{(4 \pi)^{2}}
$$

where $\beta$ is given in (5.23).

Next, we consider $\Gamma_{X^{2}}$ given in (4.21). We rewrite $\Gamma_{X^{2}}$ as follows

$$
\Gamma_{X^{2}}=\frac{1}{96} \frac{1}{(4 \pi)^{2}} \int d^{4} \theta X^{\alpha} X_{\alpha} \frac{1}{\square} D^{2}\left(p \kappa^{2} K+U\right)+\text { h.c. }
$$

where

$$
p=744
$$

and

$$
\begin{aligned}
U & =\kappa^{2} K\left(-p-\sum_{k}\left(\prod_{H} n_{k}^{H}\right)-\sum_{d}\left(\prod_{H} n_{d}^{H}\right)-n_{I}-1+\sum_{H} n_{V}^{H}+3\right) \\
& +2 \ln \operatorname{det} \mathcal{G}_{I \bar{J}}+2 \ln \mathcal{G}_{S}+2 \sum_{\text {sec }}\left(\prod_{H} n_{\text {sec }}^{H}\right) \ln \operatorname{det} \mathcal{G}_{\text {sec }}
\end{aligned}
$$


In (A.10), we have included the contribution from the gravitino, +3 [19]. The variation of $\Gamma_{X^{2}}$ under Kähler transformations (4.25) was given in (4.27) and reads

$$
\begin{aligned}
\delta_{K} \Gamma_{X^{2}} & =\frac{1}{24} \frac{1}{(4 \pi)^{2}} \int d^{2} \theta \kappa^{2} F(T, S) X^{\alpha} X_{\alpha}\left[\sum_{k}\left(\prod_{H} n_{k}^{H}\right)+\sum_{d}\left(\prod_{H} n_{d}^{H}\right)+n_{I}\right. \\
& \left.+1-\sum_{H} n_{V}^{H}-3\right]+ \text { h.c. }
\end{aligned}
$$

In (A.11), we have included the contribution from the gravitino. Inserting the information about the massless spectrum of $Z_{3}$ into (A.11) yields a non-vanishing result for the pure Kähler anomaly

$$
\delta_{K} \Gamma_{X^{2}}=\frac{1}{24} \frac{1}{(4 \pi)^{2}} 888 \int d^{2} \theta \kappa^{2} F(T, S) X^{\alpha} X_{\alpha}+\text { h.c. }
$$

In the standard (T,S)-coordinate system, it is readily seen that the dependence of $U$ on the untwisted moduli cancels out. This can be seen by comparing $U$, as given in (A.10), against $N$, as given in (A.3). Again, when supplementing $U$ with the counterterms introduced in the main part of this paper, the moduli dependence of these counterterms cancels out as well. Hence, the moduli dependence of $U$ (supplemented by counterterms) cancels out in any coordinate system. It is then tempting to conjecture that the coefficient $p$ in (A.9) represents the amount of the $K$-terms in (A.8) which is removed by the Green-Schwarz mechanism, as discussed in section 5, yielding

$$
3 \sigma+4 \tau=-\frac{744}{\beta} \frac{1}{2} \frac{1}{(4 \pi)^{2}}
$$

\section{References}

1. P. Binétruy, G. Girardi, R. Grimm and M. Müller, Phys. Lett. B189 (1987) 83; P. Binétruy, G. Girardi and R. Grimm, LAPP preprint TH-275/90.

2. E. Cremmer, B. Julia, J. Scherk, S. Ferrara, L. Girardello and P. van Nieuwenhuizen, Phys. Lett. B79 (1978) 231; Nucl. Phys. B147 (1979) 105; B. Zumino, Phys. Lett. B87 (1979) 203; E. Cremmer, S. Ferrara, L. Girardello and A. van Proyen, Phys. Lett. B116 (1982) 231; Nucl. Phys. B212 (1983) 413; E. Witten and J. Bagger, Phys. Lett. B115 (1982) 202; J. Bagger, Nucl. Phys. B211 (1983) 302. 
3. G. L. Cardoso and B. A. Ovrut, University of Pennsylvania preprint UPR-0481T (1991), to be published in Proc. Strings and Symmetries 1991, Stony Brook, May 20-25, 1991; Proc. of the International Europhysics Conference, Geneva, Switzerland, July 1991.

4. L. J. Dixon, J. A. Harvey, C. Vafa and E. Witten, Nucl. Phys. B261 (1985) 678; Nucl. Phys. B274 (1986) 285.

5. M. Cvetič, J. Louis and B. A. Ovrut, Phys. Lett. B206 (1988) 227; M. Cvetič, J. Molera and B. A. Ovrut, Phys. Rev. D40 (1989) 1140.

6. G. Moore and P. Nelson, Phys. Rev. Lett. 53 (1984) 1519; J. Bagger, D. Nemeschansky and S. Yankielowicz, Nucl. Phys. B262 (1985) 478.

7. L. J. Dixon, V. S. Kaplunovsky and J. Louis, Nucl. Phys. B355 (1991) 649.

8. G. L. Cardoso and B. A. Ovrut, Nucl. Phys. B369 (1992) 351; J. P. Derendinger, S. Ferrara, C. Kounnas and F. Zwirner, Nucl. Phys. B372 (1992) 145.

9. J. Louis, PASCOS 1991 Proceedings, P. Nath ed., World Scientific 1991

10. I. Antoniadis, K. S. Narain and T. R. Taylor, Phys. Lett. B267 (1991) 37.

11. J. Lauer, J. Mas and H. P. Nilles, Phys. Lett. B226 (1989) 251; R. Dijkgraaf, H. Verlinde and E. Verlinde, in Proc. Perspectives in String Theory, Copenhagen 1987

12. M. B. Green and J. H. Schwarz, Phys. Lett. B149 (1986) 117.

13. M. Müller, Nucl. Phys. B264 (1986) 292.

14. E. Witten, Phys. Lett. B155 (1985) 151.

15. S. Ferrara, D. Lüst, A. Shapere and S. Theisen, Phys. Lett. B225 (1989) 363;

S. Ferrara, D. Lüst and S. Theisen, Phys. Lett. B233 (1989) 147; M. Cvetič, A. Font, L. E. Ibáñez, D. Lüst and F. Quevedo, Nucl. Phys. B361 (1991) 194.

16. L. J. Dixon, V. S. Kaplunovsky and J. Louis, Nucl. Phys. B329 (1990) 27. 
17. S. L. Adler and W. Bardeen, Phys. Rev. 182 (1969) 1517.

18. R. Garreis, M. Scholl and J. Wess, Z. Phys. C28 (1985) 623.

19. L. Alvarez-Gaumé and P. Ginsparg, Annals of Phys. 161 (1985) 423.

20. S. Ferrara and M. Villasante, Phys. Lett. B186 (1987) 85; P. Binétruy, G. Girardi, R. Grimm and M. Müller, Phys. Lett. B195 (1987) 389; P. Binétruy, G. Girardi and R. Grimm, Phys. Lett. B265 (1991) 111.

21. G. Girardi and R. Grimm, Nucl. Phys. B292 (1987) 181.

22. L. E. Ibánez and D. Lüst, preprint CERN TH.6380/92; I. Antoniadis, E.Gava and K.S. Narain, preprint CPTH-A162.0392, IC/92/51(1992) 


\section{Figure Captions}

Figure 1: We present the anomalous three-point triangle graph for matter fields $\phi^{k}$ running around the triangle loop.

Figure 2: We present the anomalous tree graph contribution from the Chern-Simons and Green-Schwarz terms. 